\title{
Teatro mudo como alternativa de educação em saúde bucal com indígenas no Estado do Pará
}

\author{
Aluísio Ferreira Celestino Júnior*; Débora Alves Soeiro**; Jordana Alexandre Braz Aimée**; Rayssa \\ Martins Carvalho**; Rebeca Menezes Rendeiro**; Tamiris Faro Casseb**; Wellisson Luigi Lima \\ Pinheiro**
}

* Docente do curso de Odontologia do Centro Universitário do Pará

** Acadêmico do curso de Odontologia do Centro Universitário do Pará

Recebido em 28/12/2016. Aprovado em 23/01/2017.

\begin{abstract}
RESUMO
Dentre as habilidades e competências que envolvem a formação em Odontologia, parte delas exige grande criatividade. Na educação em saúde, esta habilidade é intensamente exigida e as atividades cênicas são oportunidades para que mudanças de comportamento ocorram no contexto de sua formação. Este estudo foi parte integrante de projeto de extensão onde foram realizadas atividades de Promoção de Saúde com etnias indígenas assistidas pela Casa de Saúde Indígena (CASAI) em BelémPará. Dentre estas atividades, os autores desenvolveram oficinas pedagógicas com os diferentes grupos, exame clínico, aplicação tópica de flúor, tratamento restaurador atraumático e, transversalmente, ações educativas com destaque para atividade cênica em sua vertente conhecida como Teatro Mudo. A diversidade linguística observada na CASAI constitui um desafio importante às estratégias de educação em saúde. Entretanto, ao se priorizar formas diferentes de comunicação como aquelas do Teatro Mudo, os limites são em grande parte superados. O lúdico com modelagem cênica positiva é um recurso extremamente rico na educação em saúde, principalmente diante de condições de limite da comunicação oral. Este recurso consegue traduzir ideias mostrando-se como tecnologia educativa viável para educação em saúde com estes grupos.

Descritores: Educação em Saúde. Saúde Bucal. Populações Indígenas.
\end{abstract}

\section{INTRODUÇÃO}

Atividades lúdicas como ferramenta de educação em saúde bucal têm sido utilizadas por meio de diferentes estratégias em todas as áreas da saúde. A Odontologia vem integrando cada vez mais o paradigma de promoção de saúde nestas estratégias, as quais potencializam maior adesão e transformação de hábitos de cuidados com a saúde bucal ${ }^{1}$.
Embora haja um declínio da incidência de cárie no mundo, esta enfermidade ainda é um grande desafio sanitário em nosso país. Houve melhora significativa nos últimos anos, mas na região amazônica, a mais prevalente patologia bucal toma proporções mais sérias que no restante do país. Tomando-se como referência o mais recente levantamento epidemiológico realizado no Brasil, verifica-se que esta é a região 
com as maiores dificuldades, apresentando o pior quadro de prevalência de cárie aos 12 anos que é a principal idade de comparação entre diferentes populações ${ }^{2}$.

Esta patologia, mesmo nesta região, não apresenta perfil uniforme em termos de distribuição na população. Este agravo se concentra principalmente em populações com perfil socioeconômico desfavorável, principalmente nas periferias das grandes cidades como também nas áreas mais interiorizadas ${ }^{3}$.

$\mathrm{Na}$ Amazônia, as transições epidemiológica e demográfica também não são homogêneas. Há lugares desta grande região brasileira onde as doenças infectocontagiosas são expoentes de adoecimento na população em relação às doenças crônico-degenerativas que também avançam. São comunidades mais vulneráveis como aquelas onde o índice de desenvolvimento humano é cronicamente crítico ${ }^{4}$.

As populações tradicionais como quilombolas, ribeirinhos e indígenas acompanham esta situação preocupante com o agravante de não se ter muitas informações epidemiológicas. Os benefícios que poderiam garantir melhor saúde bucal verificados em parte da população brasileira não é homogêneo também ${ }^{5}$.

De modo particular, as populações indígenas apresentam-se em grande parte sob uma intensa transição nutricional, em que alimentos industrializados vão substituindo a alimentação tradicional ${ }^{6}$. Desta forma, estas populações indígenas vão assumindo um novo traço de mudança na sua alimentação, passando a ser cada vez mais hipercalórica, rica em sódio e de valor nutricional precário.

Este conjunto de fatores influencia o surgimento de doenças como diabetes mellitus, hipertensão arterial, obesidade, cárie, entre outras patologias associadas ao novo estilo de vida. Concorrem para isso o fato de que estas comunidades adquirem o novo hábito de risco, mas nem sempre assumem o hábito de proteção que a ele deveria estar associado. Em relação às doenças bucais é comum observar uma mudança da dieta, que não é acompanhada do autocuidado em saúde bucal ${ }^{7}$.
Se sobrepõe a este problema, a dificuldade de se obter informações qualificadas sobre prevenção e acesso aos serviços de saúde bucal por populações indígenas ${ }^{8}$. Não é difícil supor o quanto este quadro é preocupante.

Segundo Pose $^{9}$, as mudanças e pressões exercidas pela sociedade nacional têm sido apontadas como fator determinante para o acréscimo de diferentes níveis de prevalência de cárie e outras enfermidades.

Para fazer frente a este quadro complexo é necessário investir em condições que favoreçam uma percepção de cuidado a ser tomado pelos próprios indígenas. Isto pode ser feito de diversas formas e uma delas se refere a estratégias de educação em saúde que possam informá-los e motivá-los para um comportamento de risco menor que estes supramencionados ${ }^{10}$.

Partindo desse pressuposto, os autores desenvolveram projeto de extensão universitária que integrou diversas atividades de cuidado a indígenas albergados na Casa de Saúde Indígena do Distrito Sanitário Especial Indígena GuamáTocantins. Tais cuidados tiveram como paradigma de referência a Promoção de Saúde. No contexto deste projeto foi desenvolvido um teatro educativo, em sua vertente "Teatro Mudo", com o objetivo de promover a melhor percepção de riscos, como também de cuidados de saúde bucal no sentido de orientá-los de maneira a assumirem uma postura de protagonismo destes cuidados com sua saúde.

\section{RELATO DE EXPERIÊNCIA}

Este é um relato de experiência desenvolvido a partir de vivência em uma unidade de apoio à saúde indígena na periferia da cidade de Belém, distrito de Icoaraci, em espaço institucional denominado Casa de Saúde Indígena (CASAI) do Distrito Sanitário Especial Indígena Guamá/Tocantins (DSEI GUA-TOC). Segundo a Biblioteca Virtual em Saúde ${ }^{11}$ um relato de experiência é um tipo de fonte de informação dedicada à coleta de depoimentos $\mathrm{e}$ registro de situações e casos relevantes que ocorreram durante a implementação de um programa, projeto ou em uma dada situação problema. 
A Casa de Saúde Indígena foi criada para atender a demandas de alojamento e alimentação para comunidades indígenas do Distrito Sanitário Especial Indígena Guamá-Tocantins (DSEIGUATOC) em trânsito, provenientes principalmente de comunidades indígenas do Estado do Pará, estando submetidos a regulamentação do Sistema Único de Saúde (SUS), embora receba também indígenas de outros Estados.

A CASAI de Belém fica localizada no distrito de Icoaraci, distante $21 \mathrm{~km}$ do centro da capital paraense e tem população flutuante de cerca de 125 indígenas das etnias Amanayé, Anambé, Asurini, Atikum, Guajarara, Guarani, Ka'apor, Karajá, Kaxuyana, Kayapó, Munduruku, Parakanã, Suruí, Tembé, Timbira, Tiriyó, Waiwai, Xikrin e Zoé, as quais pertencem ao Distrito Sanitário Especial Indígena.

Os indígenas que se albergam na CASAI o fazem com a finalidade de receberem suporte a tratamentos de saúde que não estão disponíveis em seus locais de origem. Este tratamento, em geral, se refere a necessidade da média e alta complexidade do Sistema Único de Saúde.

A maioria dos albergados, entretanto, não é formada por enfermos, mas por seus acompanhantes. Embora a decisão de que cada doente seja acompanhado por apenas um de seus familiares, esta norma não é rigorosamente respeitada na CASAI de Icoaraci. Por vezes, é possível encontrar o núcleo familiar completo do enfermo. Este número em alguns casos supera meia dúzia de acompanhantes.

Muitos destes acompanhantes permanecem sem muitas atividades nas instalações da CASAI e podem permanecer por longos períodos acompanhando o seu parente em tratamento.

No tempo de permanência nesta unidade de apoio, a gerência da CASAI e sua equipe realizam programações com enfoque prioritário na educação em saúde. Para este fim, foi solicitada a participação dos autores nas atividades que foram formalizadas através de projeto de extensão universitária.

O trabalho realizado foi parte integrante do projeto onde são realizadas outras atividades educativas, preventivas e curativas direcionadas à comunidade assistida pela CASAI. Dentre estas atividades os autores desenvolveram oficinas pedagógicas com os diferentes grupos, além de aplicação tópica de flúor, tratamento restaurador atraumático, exame clínico bucal para identificação de lesões com características de malignidade, lesões de cárie e doenças periodontais. Casos complexos foram encaminhados para atendimento pelos autores e outros voluntários na clínica de graduação no centro de Belém.

Paralelamente, foi realizada atividade cênica em sua vertente conhecida como Teatro Mudo, abordando os seguintes temas: importância de manter os dentes e gengivas saudáveis, técnicas de autocuidado relacionadas a escovação, uso do fio dental e orientação de dieta adequada.

$\mathrm{O}$ projeto se desenvolveu com estritas características extensionistas, ou seja, não se tratou de pesquisa científica, mas de desenvolvimento de ações já testadas pela ciência, portanto, não pretendeu gerar conhecimento generalizável, desta maneira, não havendo necessidade de submissão a um comitê de ética em pesquisa por não se configurar como pesquisa propriamente dita. A área onde as diferentes etnias se encontravam (CASAI) não é território indígena, mas do Ministério da Saúde, portanto não foi necessária autorização da FUNAI para acesso em área Indígena.

Apesar disso os autores comprometeramse em respeitar a visão de mundo das diferentes etnias presentes na CASAI no momento das visitas (costumes, estética, crenças religiosas, organização social, diferenças linguísticas e estrutura política) além de outras peculiaridades relacionadas à sua condição, sejam elas de ordem intelectual, psicológica e social.

Em todas as etapas de desenvolvimento das ações, foram rigorosamente observadas as normas vigentes relacionadas a estas populações em sua vulnerabilidade, seja de modo geral e em nível individual (enfermidade, limitação sensorial ou motora, por exemplo).

As comunidades indígenas na Amazônia são muito heterogêneas e isto se deve a diferentes fatores como distintas cosmovisões (crenças, 
valores, atitudes), relação com o ambiente, interações internas e com outros grupos étnicos, entre outros elementos ${ }^{12}$.

A relação de contato com a população não índia, por exemplo, influencia de maneira relevante estes grupos humanos. O contato inicial destas comunidades com a sociedade envolvente deu-se em momentos distintos e com intensidade também diversificada entre as diferentes etnias. Isto representou, na história destes povos, elementos de fortalecimento nas relações, de coesão interna em muitos casos, mas também de desarmonia em diferentes nuanças, significando para estes povos mudanças importantes em sua cultura, algumas delas não muito favoráveis e, eventualmente, colocando em risco não somente traços de sua cultura, mas a própria vida humana ${ }^{13}$.

Parte destas transformações culturais se deu no aspecto da linguagem. Com a expansão da comunidade não índia iniciada após a colonização europeia ainda no século XVI, um processo gradual, mas intenso fez com que algumas etnias se confrontassem com o colonizador absorvendo elementos da linguagem e por vezes, perdendo a referência da língua materna, principalmente nas novas gerações ${ }^{14}$.

Esse elemento fundamental da identidade cultural, entretanto, não foi de todo abandonado em várias populações indígenas. Preservado, mantem-se como elemento de coesão destes povos, mas por outro lado limita a comunicação com povos de língua diferente ${ }^{15}$.

Este fenômeno de diversidade linguística, fica patente na CASAI em Icoaraci. Neste espaço de cuidado, ficam albergadas diversas etnias indígenas da Amazônia que se deslocam até a CASAI em busca de cuidado com sua saúde. Parte dessas pessoas domina razoavelmente o idioma português, outros, entretanto, desconhecem completa ou parcialmente este idioma, o que requer a presença de intérpretes. Não são duas ou três línguas faladas pelas diferentes comunidades, mas um número diversificado que dificulta, por vezes, a interação entre os diferentes grupos étnicos, principalmente em atividades coletivas. Ações de atenção à saúde individuais ou que envolvam apenas uma língua nativa nem sempre representam grande dificuldade, mas nas atividades simultâneas com grupos de línguas diferentes, esta barreira se impõe.

Ao se planejar ações com esta dimensão é importante que os atores envolvidos tenham clara a dificuldade dos símbolos orais tão desconhecidos por muitos albergados da CASAI. Assim sendo, é importante planejar e implementar estratégias que contemplem as minorias linguísticas em sua diversidade.

Para solucionar parte do problema, a presença de um intérprete é certamente desejável, mas com grande limitação num contexto tão plural em termos linguísticos. Atividades cênicas, por exemplo, podem valorizar a expressão corporal com símbolos universais de comunicação do corpo. O teatro, portanto, é uma estratégia que em muito valoriza a comunicação em contextos semelhantes.

O teatro é uma invenção humana antiga, intimamente ligada ao surgimento do homem e sua necessidade de se comunicar ${ }^{16}$. A arte cênica é profundamente motivadora para crianças e adolescentes, ela os afeta nos aspectos cognitivos, emocionais, motores e sociais e como tal "demanda um alto grau de atenção e concentração, estimula memória e percepção (...) fomenta a imaginação e a expressividade ${ }^{17}$."

$\mathrm{O}$ roteiro da peça foi cuidadoso, mas permitiu eventuais improvisos para valorizar a participação da comunidade expectadora. A expressão corporal utilizada foi propositadamente exuberante, caricata às vezes para não deixar dúvidas sobre seu conteúdo. O bom humor, ao ser valorizado, dá leveza à mensagem para que seja positiva, motivadora. Elementos dramáticos (de dor, por exemplo) poderão existir, mas com soluções no contexto de cuidados com a saúde, como foi apresentado.

As personagens representadas foram seres animados (criança, jovem, mãe, pai, cirurgiõesdentistas) como também da animação fantástica (sujidades, bactéria, escovas de dentes, fio dental, creme dental, flúor, espelho clínico). Ao dar vida a estes personagens, ganha-se em dinamismo cênico ao mesmo tempo que estimula com o lúdico o envolvimento da plateia por meio 
da imaginação.

Crianças e adultos participaram desta etapa do projeto de extensão e, certamente representa uma estratégia de educação que pode ser estimulada no contexto de educação em saúde para grupos de línguas diferentes como os encontrados na CASAI como também para pessoas com deficiência auditiva, por exemplo.

Independentemente das condições mencionadas, é um recurso que poderá ser utilizado com vários grupos, mesmo aqueles que não possuem as características acima descritas.

\section{CONSIDERAÇÕES FINAIS}

O teatro mudo é uma ferramenta que, por valorizar a expressão corporal permite uma comunicação eficaz em grupos linguisticamente distintos como os encontrados na CASAI em Belém. Esta forma de arte conseguiu traduzir ideias mostrando-se como tecnologia educativa viável para educação em saúde com estes grupos.

A necessidade de comunicar ideias deve permitir uma imersão no mundo cênico de forma a transformar tais ideias em comunicação. Isto deve ser considerado por quem faz educação em saúde para diferentes grupos humanos. Os desafios não são apenas a língua, mas diversos elementos da cultura de indígenas, ribeirinhos, moradores de favelas ou de rua, trabalhadores, jovens, idosos, crianças, grávidas ou qualquer outro grupo que trazem suas complexas vivências a serem descortinadas pela linguagem expressa de forma tão plural num contexto que se faça razoavelmente compreendido.

\section{ABSTRACT \\ Silent theater as educational alternative in oral health with indigenous in Pará}

Among the skills and competencies that involve training in dentistry, some of them require great creativity. In health education, this ability is intensely required, and scenic activities are opportunities for behavioral changes to occur in the context of their training. This study was an integral part of the extension project where health promotion activities were carried out with indigenous ethnic groups assisted by Casa de Saúde Indígena/CASAI in Belém/Pará. Among these activities, the authors developed pedagogical workshops with the different groups, clinical examination, topical application of fluoride, atraumatic restorative treatment and, transversally, educational actions with emphasis on scenic activity in its aspect known as Silent theater. The linguistic diversity observed in CASAI is an important challenge to health education strategies. However, by prioritizing different forms of communication such as those in the Silent theater, the limits are largely overcome. The playful with positive scenic modeling is an extremely rich resource in health education, especially in the face of limited oral communication conditions. This resource can translate ideas showing as viable educational technology for health education with these groups.

Descriptors: Health Education. Oral Health. Indigenous Population.

\section{REFERÊNCIAS}

1. Oliveira JCC. Atividades lúdicas na odontopediatria: uma breve revisão da literatura. Rev Bras Odontol. 2014; 7(1):103-7.

2. Brasil. Ministério da Saúde - Secretaria de Atenção à Saúde - Departamento de Atenção Básica. Pesquisa nacional de saúde bucal 2010. Brasília, 2010.

3. Roncalli AG, Tsakos G, Sheiham A, Souza GC, Richard G, Watt RG. Social determinants of dental treatment needs in brazilian adults. BMC Public Health. 2014; 14:1097. DOI:10.1186/1471-2458-14-1097.

4. Programa das Nações Unidas para o Desenvolvimento - PNUD: Atlas do desenvolvimento humano dos municípios brasileiros, 2015. [Acesso em: 27 jul 2016]. Disponível em: http://www.pnud.org.br/ $\underline{\text { IDH/Default.aspx } \text { ?indiceAccordion }=1 \& l i=1}$ i_AtlasMunicipios

5. Antunes JLF. Intervenções em saúde pública e seu impacto nas desigualdades sociais em saúde, Tempo Social. 2015; 27(1):161-74.

6. Queiroz SSMP, Xavier KO. Práticas alimentares do grupo indígena Kariri-xocó, de Lauro de Freitas-BA: sustentabilidade e 
desafios em contextos de mudanças. Food Nutr Health. 2015;10(3):649-62.

7. Moura PG, Batista LRV, Moreira EAM. População indígena: uma reflexão sobre a influência da civilização urbana no estado nutricional e na saúde bucal. Rev Nutr PUCCAMP. 2010;23(3):459-65.

8. Sá CS, Kuhnen M, Santos IF, Arruda MP, Toassi RFC. Planejamento em saúde bucal na atenção primária à saúde: da teoria à prática. Rev APS. 2015;18(1):92-101.

9. POSE SB. Avaliaçäo das condiçöes de saúde bucal dos índios Xavánte do Brasil Central [dissertação], Rio de Janeiro: Escola Nacional de Saúde Pública;1993.

10. Souza MEM, Carvalho ES, Fernandes ABSP, Carvalho FK, Carvalhal CO, Dutra ALT. A educação em saúde como medida de prevenção e promoção da saúde bucal. Full Dent Sci. 2015;6(22):239-48.

11. Biblioteca Virtual em Saúde (BVS). Relato de Experiência; 2016. [Acesso em: 31 mai 2016]. Disponível em: http://brasil.bvs.br/ relatosexperiencia

12. Resende LP, Rodrigues L; Menegócio AM. A realidade da tuberculose nos indígenas brasileiros com diversidade de etnias em menores de 15 anos de idade. Ensaios Cienc. 2014;18(2)105-11.
13. Tagliari IA, Ferreira MB, Rocha SLR, Pizzi J, Leite N. Excesso de peso e baixa estatura em crianças de comunidades urbana, rural e indígena. Rev Bras Crescimento Desenvolv Hum. 2016;26(1), 67-73.

14. Silva MNF, Castilho ACC, Chaves ACA, Pereira C, Maciel, RF, AlcântaraMM, et al. Diversidade linguística no Pará: mundos de línguas indígenas e de língua portuguesa. Rev Margens Interdisc. 2014 8(10):97-108.

15. Braga AO, Bastos ACP. O português do posto indígena Guaporé: breve história do contato linguístico. Moara. 1998;1(3):46-50.

16. Silveira AFM. O resgate social através do teatro. 2016. [Acesso em: 02 jun 2016]. Disponível em: http://www.avm.edu.br/doc pdf/monografias_publicadas/C203477.pdf

17. Margoni BMF. A arte cênica como instrumento para o desenvolvimento cognitivo da criança. 2016. [Acesso em: 02 ago 2016]. Disponível em: http://educare ceunsp.net/revista/artigos/no2/artigo_03

Correspondência para:

Aluísio Ferreira Celestino Júnior

e-mail: celestinojr@yahoo.com.br

Av. Governador José Malcher, 163/305-A

66035065 - Nazaré - Belém/Pará 